\title{
Hepatitis A outbreak predominantly affecting men who have sex with men in Northern Ireland, October 2008 to July 2009
}

O Sfetcu (Otilia.Sfetcu@ecdc.europa.eu) ${ }^{1,2}$, N Irvine' ${ }^{1}$, S L Ngui ${ }^{3}$, C Emerson ${ }^{4}$, C McCaughey5 P Donaghy $^{1}$

1. Health Protection Service, Public Health Agency, Northern Ireland, United Kingdom

2. European Programme for Intervention Epidemiology Training (EPIET), European Centre for Disease Prevention and Control $(E C D C)$, Stockholm, Sweden

3. Virus Reference Department (VRD), Health Protection Agency, London, United Kingdom

4. Genito-Urinary Medicine Clinic, Royal Hospitals, Belfast HSC Trust, Northern Ireland, United Kingdom

5. Regional Virus Laboratory, Royal Hospitals, Belfast HSC Trust, Northern Ireland, United Kingdom

Sfetcu O, Irvine N, Ngui SL, Emerson C, McCaughey C, Donaghy P. Hepatitis A outbreak predominantly affecting men who have sex with men in Northern Ireland, October 2008 to July 2009. Euro Surveill. 2011;16(9):pii=19808. Available online: http://www.eurosurveillance.org/ViewArticle.aspx?Articleld=19808

We describe an outbreak of hepatitis A which evolved in Northern Ireland between October 2008 and July 2009 , against a background of large concurrent hepatitis A outbreaks in various parts of Europe. Thirty-eight cases were defined as outbreak cases using a stratified case definition; 36 were males with a median age of 29 years and of the 28 males whose sexual orientation was known, 26 were men who have sex with men (MSM). Detailed descriptive epidemiology data collected through standardised questionnaires, together with sequencing of a 289 bp fragment of the VP1/2PA region of the virus, significantly aided the understanding of the spread of the outbreak when non-MSM cases occurred. The sequence of the outbreak strain, genotype IA, was indistinguishable from that involved in a large outbreak in the Czech Republic. Although seeded in a generally susceptible Northern Ireland population, the outbreak remained mostly contained in MSM, showing this sub-population to be the most vulnerable despite ongoing hepatitis $A$ vaccination programmes in genito-urinary medicine clinics.

\section{Introduction}

Hepatitis $A$ is an acute, usually self-limiting disease caused by infection with the hepatitis A virus (HAV) [1]. Transmission is usually by the faecal-oral route, including person-to-person spread, contaminated water or food products, but has also been associated with outbreaks in injecting drug users and men who have sex with men (MSM) [2-4]. The mean incubation period is 28 days (range 15-50 days). Asymptomatic and mild disease is common in children but the majority of adults who become infected are symptomatic, often with acute jaundice [5]. With increasing hygiene in developed countries, hepatitis $A$ infection has become much less common leading to populations with a large majority of people who are not immune. One consequence of this is that proportionately more susceptible adults are infected when outbreaks do occur, and consequently more severe clinical symptoms are seen [6]. Peak infectivity occurs two weeks before onset of jaundice and falls rapidly thereafter. The diagnosis is made by serological detection of anti-HAV IgM which becomes detectable at the onset of symptoms and can persist for up to six months. HAV RNA is also a marker of acute infection and is present in the patient's serum prior to the appearance of anti-HAV IgM [7]. In regions with a low incidence of HAV infection, sequencing of HAV RNA can be used to link apparently sporadic cases and outbreaks [8].

Monovalent and combined hepatitis A vaccines have been available since 1995 and are all highly immunogenic. Nearly $100 \%$ of adults will develop protective levels of antibody within one month after a single dose of vaccine [9]. In the United Kingdom (UK), preexposure vaccination is recommended for selected risk groups, including MSM who have multiple partners, particularly during periods when outbreaks are occurring $[5,10]$. Hepatitis A vaccine alone or combined with human normal immunoglobulin can be effectively used in preventing infections in contacts of cases, if administered within 14 days of the onset of symptoms in the index case [5]. Since 2006, MSM attending the healthcare services specialised in sexually transmitted infections, the genito-urinary medicine (GUM) clinics, in Northern Ireland have been offered combined hepatitis $A$ and $B$ vaccination, in line with guidance from the British Association of Sexual Health and HIV-Clinical Effectiveness Group (UK BASHH) [10]. However, vaccination coverage among MSM is not known.

Hepatitis A is a notifiable disease in Northern Ireland (population 1,775,000). Patients presenting with clinical symptoms which are confirmed by the Regional Virology Laboratory are reported to the regional surveillance unit of the Public Health Agency. Since 2003, there have been between zero and seven sporadic cases 
reported annually (Figure 1). After a decade of decrease of hepatitis $A$ incidence in Europe an outbreak began in the autumn of 2007 in Latvian injecting drug users followed by spread into the general population and lasted until the end of 2008 [11]. In autumn 2008, the Czech and Slovak Republics reported large outbreaks initially in injecting drug users and Roma communities respectively, followed by community spread within the general population $[12,13]$. Subsequently outbreaks among MSM have been reported from Spain (Barcelona) [14] and Italy (Rome) [15].

In October 2008, two hepatitis A cases were reported in Northern Ireland, followed by another four in November after a median time interval of 30 days. All cases were in MSM, aged between 25 and 40 years. An outbreak control team was convened in December 2008 to coordinate outbreak investigations and to undertake control measures. An urgent letter was issued to alert healthcare providers. The aim of the epidemiological study was to describe the course of the outbreak and identify any possible common exposures for further investigation.

\section{Methods}

Epidemiological investigation

Initially, an outbreak case was defined as any resident of Northern Ireland who tested positive for antiHAV IgM after 1 October 2008. In January 2009 cases began to occur in heterosexual males and females. At this time the outbreak control team asked for all positive samples to be sequenced in order to understand the progress of the outbreak which transcended the MSM population. Unfortunately, as sequencing of antiHAV IgM-positive samples was not a routine activity at the time, a number of samples had insufficient volume for sequencing and others had been discarded. After sequencing information became available a stratified case definition was used to describe the outbreak. A possible outbreak case was classified as a resident of Northern Ireland who tested positive for anti-HAV IgM

\section{FIGURE 1}

Laboratory reports of hepatitis A, Northern Ireland, 1998-2009 $(\mathrm{n}=241)$

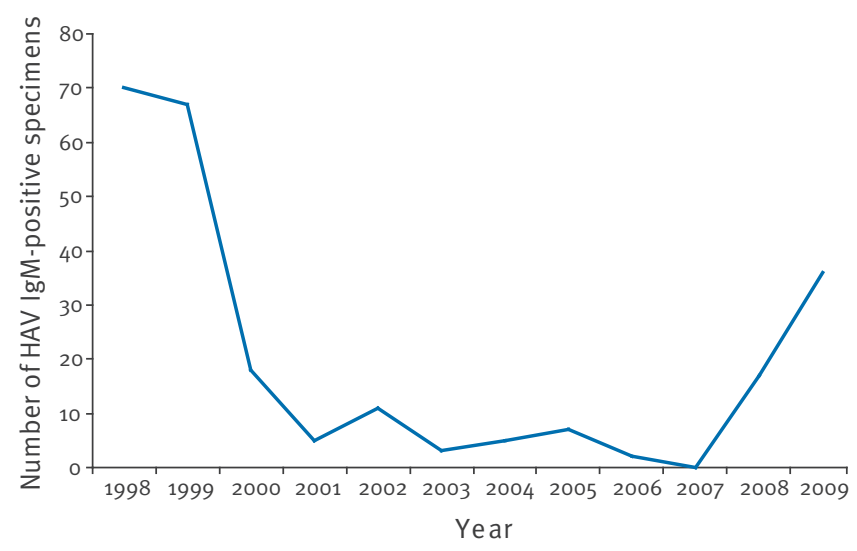

HAV: hepatitis A virus; IgM: immunglobulin M. after 1 October 2008. A possible case was upgraded to a confirmed outbreak case if the case was found after sequencing to have the genotype IA outbreak strain (referred to hereafter as the outbreak strain). Any case found to be epidemiologically linked to a confirmed case was defined as a probable outbreak case. A case was considered to be epidemiologically linked if they had been sharing the same household or had been in intimate contact with a confirmed case in the two months before becoming ill. A case was defined as sporadic if any non-outbreak strain was identified in an anti-HAV IgM-positive resident of Northern Ireland during the study period.

A standardised questionnaire administered by environmental health officers, part $A$, was used to collect the patients' demographic and clinical characteristics (age, sex, sexual orientation, occupation, place of residence, nationality, symptoms, onset date, and hospitalisation status), as well as details of selected food exposures (consumption of shellfish, raw salad, take-away food or eating outside the home), travel abroad, and contact with a symptomatic hepatitis case in the two months before onset of illness. Part B of the standardised questionnaire was administered by GUM specialists and used to collect details of sexual orientation, number of sexual partners, condom use, visiting and/ or having sex in gay venues, and other sexual behaviours, in the two months prior to onset of symptoms. Diagnoses of new and past co-infections were also collected. The questionnaires were collated at the Public Health Agency surveillance unit and data entered into an enhanced surveillance database and STATA (Stata Statistical Software: Release 10, StataCorp 2007) was used to produce descriptive statistics.

Virological analysis

Serological diagnosis was performed by the regional virology laboratory at Royal Victoria Hospital in Belfast using the Abbott Architect anti-HAV IgM assay. Where residual sample volume was available this was forwarded to the virus reference department (VRD) of the Health Protection Agency for HAV RNA detection and sequencing. The HAV RNA was extracted from serum and reverse-transcribed into cDNA by random hexamers. Amplification of the VP1/2PA junction was performed by nested PCR as described previously [16] using primers $\mathrm{HAV}_{6}, \mathrm{HAV}_{7}$ in this reference for primary amplification, and BR-9 and BR-5 for secondary amplification. The PCR products were sequenced, resulting in a 289 bp sequence, and genotype assignment was preformed by alignment and comparison with sequences of known genotype in MegAlign (DNASTAR) followed by confirmation using BLAST (http://www.ncbi.nlm.nih. gov/blast/).

\section{Results}

Descriptive epidemiology

From 4 October 2008 to 1 July 2009 , a total of 43 acute hepatitis A cases were reported; of those, 38 were classified as outbreak cases and five as sporadic cases. 
Twenty-eight of the 43 cases occurred between week 502008 and week 152009 (Figure 2).

\section{Outbreak cases $(n=38)$}

Of the 38 outbreak cases, 24 met the case definition for a confirmed outbreak case, 10 cases were defined as possible and four were classified as probable. The basis for the classification of the three of the four probable cases was that they were MSM who had household contact with confirmed cases. The fourth probable outbreak case was a man whose female sexual partner was found to have the outbreak strain.

Thirty-six cases were male, with a median age of 29 years (range: 18-51 years). Information on sexual orientation was available for 28 males, 26 of whom were MSM. All ten possible cases were MSM. With the exception of the index case who was from another European country, all other cases were British or Irish citizens. Of the 33 cases for whom occupational details were available, eight were food-handlers and two were healthcare workers. The eight food-handlers worked in eight different retail premises none of which were recognised gay venues. Information on clinical symptoms was available for 31 patients, 30 of whom presented with jaundice. Other symptoms were nausea $(n=21)$, loss of appetite $(n=17)$, abdominal pain $(n=16)$, fatigue $(n=14)$, and fever $(n=13)$. Twenty-five cases were admitted to hospital.

Food history was recorded for 18 cases. No common food product, take-away or other catering establishments was identified. None of the eight premises where the food-handling cases worked during their incubation period was named by any other case.

Seven of the 31 cases with known travel history reported travel abroad in the two months prior to illness. Two MSM cases travelled in the four weeks prior to onset of symptoms, one to Prague, Czech Republic, and one to Dublin, Republic of Ireland. The other five cases travelled within the UK, two of them in the two weeks before the onset of symptoms.

Twenty of the outbreak cases, all males, attended a GUM clinic where they completed a standardised questionnaire and were screened for coinfections. Eight cases, all MSM, were diagnosed with coinfections on that occasion: three with syphilis, one with human immunodeficiency virus (HIV), one with HIV and gonorrhoea, and three with non-specific urethritis.

Data on sexual behaviour in the two months prior to onset of illness was only available for 18 of the male cases who attended GUM services. Twelve reported having sex with another male in the two months before illness; the median number of partners was one (range 1-3). Of the 12 male cases who reported having anal sex with another man, only six declared consistent condom use ('always'). Of the remaining six males reporting no sexual contact in the two-month period, three had visited gay clubs during that time.

Information on history of hepatitis A vaccination was not available for the cases.

Sporadic cases $(\mathbf{n}=5)$

From 14 October 2008 to 8 June 2009, five sporadic cases were reported. Three cases were male, aged 20-49 years, and two female, aged 20-24 years. Two were MSM whose dates of onset were separated by an interval of six months, and who reported travel to Budapest, Hungary, and Barcelona, Spain, in the two months prior to onset of illness. The third male case declared only shellfish consumption as a risk factor. Two female cases reported travel to England and Africa during the incubation period.

\section{Virology}

Twenty-nine of the 43 anti-HAV IgM-positive specimens were available for HAV RNA detection and sequencing. Sequences of the 289 bp VP1/2PA fragment derived from 23 of these samples were indistinguishable from

\section{FIGURE 2}

Hepatitis A cases by specimen date and genotype, Northern Ireland, 4 October 2008-1 July 2009 (n=43)

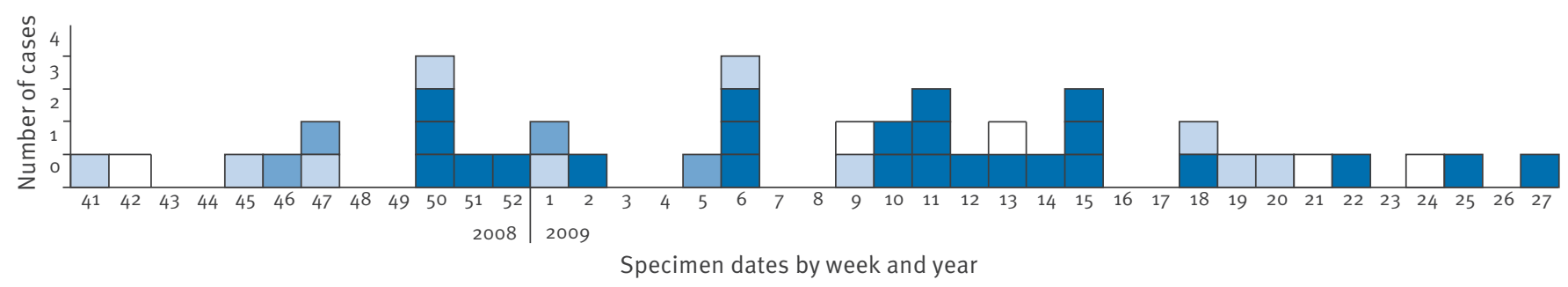

Confirmed outbreak cases: anti-HAV IgM-positive, the outbreak IA genotype $(n=24)$

$\square$ Probable outbreak cases: anti-HAV IgM-positive, epidemiologically linked, genotype not available ( $\mathrm{n=4}$ )

$\square$ Possible outbreak cases: anti-HAV IgM-positive, genotype not available $(n=10)$

$\square$ Sporadic cases: anti-HAV IgM positive, different genotypes $(n=5)$

HAV: hepatitis A virus; IgM: immunglobulin $M$. 
each other and of genotype IA; this was designated as the outbreak strain. A further sample had a $99.7 \%$ homology (one mutation in the $289 \mathrm{bp}$ fragment) to the outbreak strain. Given the low incidence of HAV in Northern Ireland and the common risk of MSM we took this to be an indication that this strain was related to the outbreak. The outbreak strain was unlike any strain previously sequenced at the VRD. Comparison of the outbreak strain with other strains isolated from recent outbreaks in Europe found it to be indistinguishable over 289 bp from the strain involved in the outbreak in the Czech Republic in 2008 (kindly provided by $\mathrm{H}$ Vennema).

Viruses isolated from two non-outbreak MSM cases shared the same sequence; the two isolates belonged to genotype IA but had only $96.9 \%$ homology to the outbreak strain. This strain had been observed at the VRD in other sporadic cases but these had all occurred FIGURE 3

Hepatitis A outbreak in Northern Ireland $(n=40)$, the source outbreak in the Czech Republic $(n=1,580)$ and the concurrent outbreak in Barcelona ( $\mathrm{n}=122), 2008-2009$

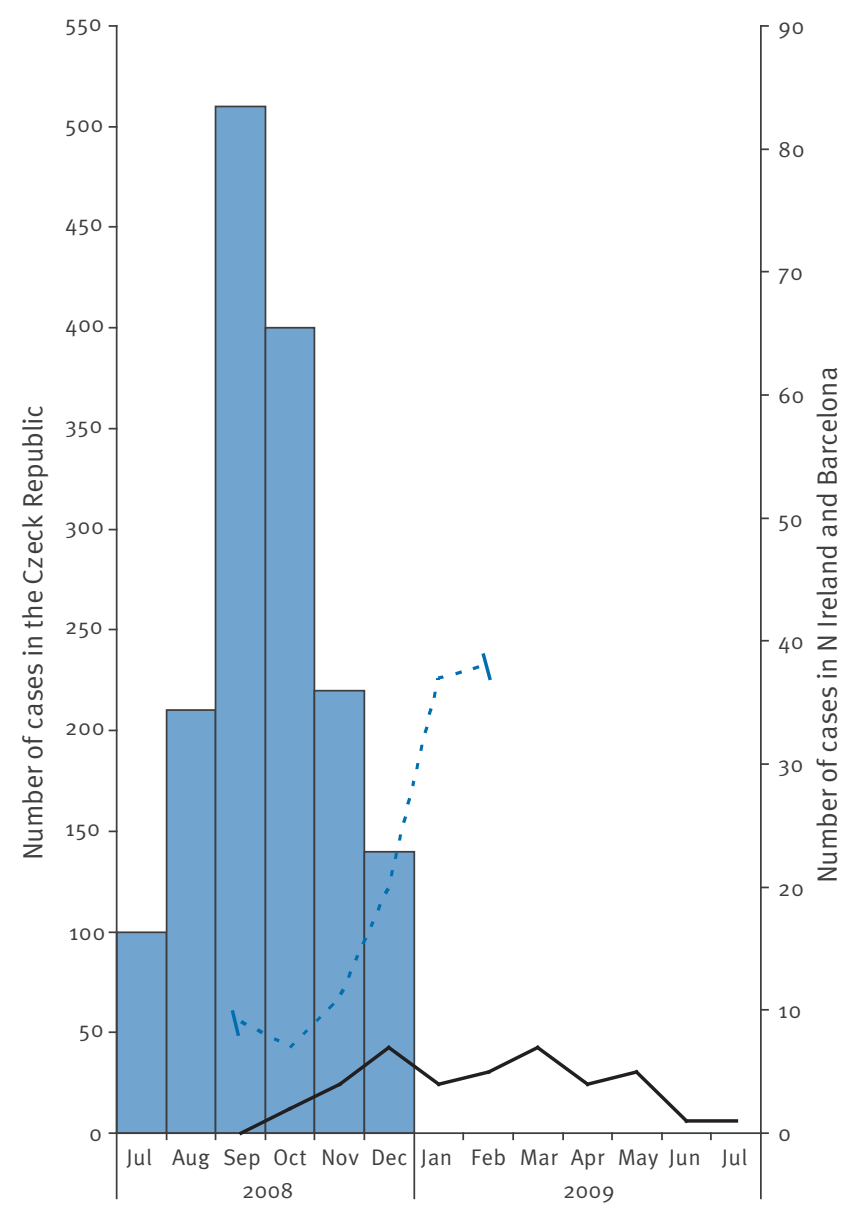

$\square$ Outbreak in the Czech Republic, genotype IA, July-December 2008

- - - Outbreak in Barcelona, genotype IA (distinct sequence), September 2008-March 2009

- Outbreak in Northern Ireland, genotype IA (Czech sequence $n=38$, Barcelona sequence $n=2$ ), October 2008 -July 2009

Source of data for the cases in the Czech Republic and Barcelona: graphs published in $[9,12]$. in mainland England. Comparison of this sequence with other European outbreak strains found it to be indistinguishable from the strain involved in the MSM community in Barcelona, in 2008 and 2009 (kindly provided by $R$ Pinto).

The remaining three samples sequenced were genotype IB and were all distinguishable from each other. The homologies of these strains to the outbreak strain were $91.7 \%, 90.3 \%$ and $84.1 \%$.

\section{Control measures}

Each case was visited at home or interviewed by telephone by the environmental health officers, in order to identify any environmental risk factors and exposures and to trace close contacts. All cases were referred to GUM clinics and their family and sexual contacts were referred to the general practitioner or to the GUM clinic to receive hepatitis $A$ vaccine and human normal immunoglobulin as appropriate. In cases known to be food handlers a risk assessment of the food premise was conducted and preventive information was provided. Outreach activities were undertaken in two of the most frequented gay venues in Belfast over a period of two months: hepatitis vaccines were offered according to personal history, and testing was offered for HIV, syphilis, hepatitis and chlamydia infection. Information on hepatitis $A$ and safe sex were distributed in gay venues with the assistance of 'Rainbow' Sexual Health Project volunteers using fliers and posters. Information on the outbreak and details on hepatitis A clinical presentation and prevention were posted on their web-site (http://www.rainbow-project.org).

\section{Discussion}

This is the first outbreak of hepatitis A in Northern Ireland following a decade of low incidence. It occurred against a background of large concurrent European outbreaks all reported to have started in at-risk groups followed by spread into the general population (Figure 3) $[2-4,10,12,13]$. The index case became ill after having travelled to Prague in September 2008, when the Czech outbreak was reaching its peak incidence [12]. Comparison of the HAV RNA sequence derived from this outbreak with that of the Czech virus appears to confirm an association.

Contrary to the experience with other European outbreaks occurring in 2008-2009 in the Czech Republic, Latvia and Slovakia, the outbreak reported here remained largely confined to the MSM sub-population, despite the majority of the general population in Northern Ireland being susceptible to HAV infection [17]. The potential for spread to the community was highlighted by the fact that cases occurred in food handlers and healthcare workers. The eight food handlers in particular had the potential to disseminate the virus widely given that they worked at eight different premises. Prompt public health action through contact tracing and post exposure prophylaxis, publicity within the gay community and the provision of 
outreach sessions at selected implicated venues have contributed to the apparent control of the outbreak. Collaboration between public health, environmental health, GUM specialists and the voluntary sector was essential.

Our study did not allow an in-depth ascertainment of transmission pathways, given that less than half of the outbreak cases returned the questionnaires on sexual risk behaviour. Similarly we were unable to document a specific venue associated with this outbreak. Notably, three MSM cases reported they had not had sex in the two month prior to illness but had visited gay venues. This raises the possibility that transmission may have occurred through environmental contamination, a finding also noted in other outbreaks [18]. Transmission via food does not seem likely as no common food source or catering venue (among food handler cases or subsequent cases) was identified.

UK national policy recognises that MSM are a vulnerable group for hepatitis $A$ and recommends they are offered pre-exposure vaccination at times of ongoing outbreaks [5]. Our experience shows this is important even in a region of low incidence, given the potential for rapid seeding of outbreaks from elsewhere.

This is further emphasised by the finding of two sporadic MSM cases during this outbreak with a common sequence indistinguishable from that implicated in the large MSM outbreak in Barcelona. That neither of them seeded an outbreak suggests either that they did not have sex in Northern Ireland while infectious (probable explanation for the case in October 2008) or that the preventive control measures were already effective and the MSM community alerted (probable explanation for the case in May 2009).

Although sequencing of HAV RNA did not significantly aid the management of the outbreak, it enabled us to define the sporadic cases and demonstrate that the outbreak was not spreading to the general community. In this setting of low HAV incidence, HAV sequencing was a valuable tool in linking apparently unlinked cases to the outbreak. Sequencing results derived from the outbreak enabled us to link the outbreak in Northern Ireland to the larger outbreak in the Czech Republic and to show that two sporadic cases, only one of which had been to Spain, shared the same strain circulating in the MSM outbreak in Spain. Comparison of the sequences from these two large outbreaks was only possible thanks to the sharing of information from the laboratories that performed the sequencing because at the time, there was no specific database for HAV sequences in the public domain.

\section{Conclusion}

This outbreak highlights the importance of timely diagnosis and reporting of cases to allow for appropriate and targeted control measures. The high rate of other sexually transmitted infections (STI) among MSM cases confirms the worth of STI testing during such an outbreak. Awareness of the value and availability of hepatitis A vaccination among MSM in Northern Ireland must be increased. Sequencing the virus and collaborating with other countries involved in similar epidemics helped us understand how this outbreak spread; sequencing of all HAV cases has continued since this time. It has subsequently come to light that HAV sequences can be sent to the Food-borne Viruses in Europe (FBVE) network for comparison with their database [19]: this network is currently making its HAV sequence database publically available.

\section{Acknowledgements}

The authors acknowledge the valuable contribution of all the Outbreak Control Team members, Environmental Health Officers, Genito-Urinary Clinic staff, and The "Rainbow "Project volunteers. Special thanks are expressed to Dr Vratislav Nemecek, Dr Harry Vennema and Dr Rosa Pinto for enabling the comparison of sequences from the Czech and Spanish outbreaks respectively.

\section{References}

1. Mandell G, Douglas, Bennett J. Principles and Practice of Infectious Diseases 7th edition. Chapter 173. United Kingdom: Churchill Livingstone; 2009

2. Payne L, Coulombier D. Hepatitis A in the European Union: responding to challenges related to new epidemiological patterns. Euro Surveill. 2009;14(3):pii=19101. Available from: http://www.eurosurveillance.org/ViewArticle. aspx?Articleld $=19101$

3. European Centre for Disease Prevention and Control (ECDC). Technical meeting on hepatitis A outbreak response, Riga, November 2008. Stockholm:ECDC. 2009. Available from: http://ecdc.europa.eu/en/publications/Publications/0811 MER_Hepatitis_A_Outbreak_Response.pdf

4. Urbanus AT, van Houdt R, van de Laar TJ, Coutinho RA. Viral hepatitis among men who have sex with men, epidemiology and public health consequences. Euro Surveill. 2009;14(47):pii=19421. Available from: http://www. eurosurveillance.org/ViewArticle.aspx?Articleld=19421

5. United Kingdom Department of Health (DH). Immunisation Against Infectious Disease, 2006. Hepatitis A. London:DH. [Accessed 9 February 2011]. Available from: http://www. dh.gov.uk/prod_consum_dh/groups/dh_digitalassets/@dh/@ en/documents/digitalasset/dh_124297.p df

6. Jacobsen $\mathrm{KH}$, Wiersma S. Hepatitis A virus seroprevalence by age and world region, 1990 and 2005 . Vaccine. 2010;28(41):6653-7.

7. Nainan OV, Xia G, Vaughan G, Margolis HS. Diagnosis of hepatitis A virus infection: a molecular approach. Clin Microbiol Rev. 2006;19(1):63-79.

8. Ngui SL, Granerod J, Jewes LA, Crowcroft NS, Teo CG, 2002 Hepatitis A Outbreaks Investigation Network. Outbreaks of hepatitis A in England and Wales associated with two co-circulating hepatitis A virus strains. J Med Virol. 2008;80(7):1181-8.

9. Plotkin SA, Orenstein WA, Offit PA, editors. Vaccines, 5 th Edition. Elsevier Inc; 2008

10. British Association of Sexual Health and HIV. Clinical Effectiveness Group. United Kingdom National Guideline on the Management of the Viral Hepatitides A, B \& C. 2008. Available from: http://www.bashh.org/documents/1927

11. Perevoščikovs J, Lucenko I, Magone S, Brila A, Curikova J, Vennema H. Community-wide outbreak of hepatitis A in Latvia in 2008 - an update. Euro Surveill. 2009;14(3): pii=19092. Available from: http://www.eurosurveillance.org/ViewArticle. aspx?Articleld $=19092$

12. Cástková J, Beneš C. Increase in hepatitis A cases in the Czech Republic in 2008 - an update. Euro Surveill. 2009;14(3):pii=19091. Available from: http://www. eurosurveillance.org/ViewArticle.aspx?Articleld=19091 
13. Hrivniaková L, Sláčiková M, Kolcunová S. Hepatitis A outbreak in a Roma village in eastern Slovakia, August-November 2008 . Euro Surveill. 2009;14(3):pii=19093. Available from: http:// www.eurosurveillance.org/ViewArticle.aspx?Articleld=19093

14. Tortajada C, de Olalla PG, Pinto RM, Bosch A, Caylà J.

Outbreak of hepatitis $A$ among men who have sex with men in Barcelona, Spain, September 2008 - March 2009. Euro Surveill. 2009;14(15):pii=19175. Available from: http://www. eurosurveillance.org/ViewArticle.aspx?Articleld=19175

15. Capobianchi MR. Large long-lasting monophyletic outbreak of Hepatitis A occurred in 2008-2009 in Rome, Italy, involving HIV-infected men who have sex with men. ESCAIDE, Stockholm. 2009; poster presentation.

16. Stene-Johansen K, Tjon G, Schreier E, Bremer V, Bruisten S, Ngui SL, et al. Molecular epidemiological studies show that hepatitis $A$ virus is endemic among active homosexual men in Europe. J Med Virol. 2007;79(4):356-65.

17. Health Protection Agency (HPA). Guidance for the Prevention and Control of Hepatitis A Infection. London:HPA. Nov 2009. Available from: http://www.hpa.org.uk/web/HPAwebFile/ HPAweb_C/1259152095231

18. Reintjes R, Bosman A, de Zwart O, Stevens M, van der Knaap $L$, van den Hoek K. Outbreak of hepatitis A in Rotterdam associated with visits to 'darkrooms' in gay bars. Commun Dis Public Health. 1999;2(1):43-6.

19. Petrignani M, Harms M, Verhoef L, van Hunen R, Swaan C, van Steenbergen J, et al. Update: A food-borne outbreak of hepatitis $A$ in the Netherlands related to semi-dried tomatoes in oil, January-February 2010. Euro Surveill. 2010;15(20):pii=19572. Available from: http://www. eurosurveillance.org/ViewArticle.aspx?Articleld=19572 